\title{
Experimental Indication of Structural Heterogeneities in Fragile Hydrogen-bonded Liquids
}

\author{
D. MORINEAU, C. ALBA-SIMIONESCO \\ CPMA URA 1104 CNRS, bâtiment 490, Université Paris-Sud, 91405 Orsay, France \\ M.-C. BELLISSENT-FUNEL \\ Laboratoire Léon Brillouin, C.E.N. Saclay 91 Gif sur Yvette, France \\ M.-F. LAUTHIÉ \\ L.A.S.I.R., 94 Thiais, France
}

September 3, 2018

\begin{abstract}
We present the first experimental characterization in molecular fragile glassformers of a "prepeak" that appears significantly below the main peak of the static structure factor. The temperature and density dependences of this prepeak are studied via elastic neutron scattering experiments under high pressure (up to $300 \mathrm{MPa}$ ) in m-toluidine and $\mathrm{m}$-fluoroaniline. The prepeak intensity increases with decreasing temperature, but it remains constant with increasing pressure while its position and width stay roughly the same. These features are opposite to those observed for the "first sharp diffraction peak" of network glasses. The origin of the phenomenon is analyzed with the help of Monte Carlo simulations. We associate the prepeak with hydrogenbond-induced heterogeneities (or clusters) whose limited size results from an exclusion effect between benzene rings that prevents the extension of a hydrogen-bond network. Implications for the dynamics of the liquid close to the glass transition are finally considered.

PACS : 64.70.Pf ; 61.25.Em.
\end{abstract}


Analyzing the structure of glasses and supercooled liquids can give some insight into the mechanisms involved in the glass transition at a micro-or mesoscopic scale. Recently there has been growing experimental evidence of the existence of heterogeneities that develop with decreasing temperature in fragile supercooled liquids [1]. Such heterogeneities are at the basis of many theories [2] of the dramatic slowing down of the relaxations when approaching the glass transition. Heterogeneities of some sort have also been invoked to explain the apparently anomalous relaxational properties of monohydric alcohols and supercooled water : these latter would be due to a clustering phenomenon involving hydrogen-bonding interactions [3]. However, the evidence for heterogeneities, domains or clusters is mostly indirect, i.e. extracted from dynamical measurements. It would thus be of major interest to find the signature of such entities directly in structural data.

Network-forming glasses and melts show a first sharp diffraction peak (FSDP) in X-ray and neutron diffraction data which, although still debated, seems convincingly attributed to some intermediate-range order [4. As for molecular systems, few results about the structure of their supercooled liquid and glassy states have been reported, and the typical neutron or X-ray pattern consists of broad peaks that evolve continuously with temperature and display no drastic changes at the glass temperature $T_{g}$. A recent low-angle neutron diffraction study has concluded to the absence of heterogeneities in deuterated propylene glycol [5].

In this letter we present the first experimental characterization for molecular fragile glassformers of the temperature and density dependence of a "prepeak" which appears at a wave vector $Q_{p p} \approx 0.5 \AA^{-1}$, i.e., significantly below the main peak of the static structure factor $S(Q)$. We focus on a series of substituted aromatic liquids, toluene (TOL), m-toluidine (MTOL) and m-fluoroaniline (MFA). An interesting property of these liquids is that crystallization can be readily avoided so that they are good candidates for a thorough study of the metastable liquid state. Despite the differences in their dipole moment and intermolecular H-bonds strength, these liquids present the same thermodynamic signature at $T_{g}$ and are considered as some of the most fragile systems [6].

The experiments were carried out on the 7C2 and G6.1 spectrometers at the reactor Orphée of the laboratoire Léon Brillouin円 (Saclay, France) at three different incident wave lengths in order to cover a large $Q$-range from 0.1 to $10 \AA^{-1}$. An isotopic purity of the samples of more than $99 \%$ was obtained by catalytic $\mathrm{H}-\mathrm{D}$ exchange for the fully deuterated MTOL $\left(\mathrm{C}_{6} \mathrm{D}_{4} \mathrm{CD}_{3} \mathrm{ND}_{2}\right)$, the partially deuterated MTOL $\left(\mathrm{C}_{6} \mathrm{D}_{4} \mathrm{CD}_{3} \mathrm{NH}_{2}\right)$, and MFA $\left(\mathrm{C}_{6} \mathrm{HD}_{3} \mathrm{FND}_{2}\right)$.

\footnotetext{
${ }^{1}$ laboratoire commun CEA-CNRS
} 
The temperature range was from $320 \mathrm{~K}$ to $77 \mathrm{~K}$, from well above the melting temperature $T_{m}$ down to well below $T_{g}$, and the pressure range was from normal pressure up to $300 \mathrm{MPa}$. The pressure was measured out of the cell and in the sample at the bottom of the cell, thus allowing to check that the system is hydrostatically compressed. Spectra were corrected for detector efficiencies, background, cell contribution and multiple scattering using standard procedures. After normalization, Placzek corrections were evaluated with a polynomial function. Other details on the experimental setup and data reduction will be given in a forthcoming publication [7].

Fully corrected static structure factors $S(Q)$ are presented in figs. 1 and 2 for MFA and partially deuterated MTOL, respectively. Like many aromatic liquids, a main diffraction peak is observed in the Q-range between $1.3 \AA^{-1}$ and $2.0 \AA^{-1}$ and present differences in shape that are mainly due to different chemical constitution and cancellation effects in the linear combination of the partial structure factors.

The most noticeable feature in the $S(Q)$ is a "prepeak" that appears at a much lower $Q$ than the main peak for MFA and MTOL (figs. 1 and 2). Its intensity increases when $T$ decreases down to $T_{g}$, at which point it stays roughly constant (cf. for instance the inset in fig. 2). As illustrated in fig. 2, the position and the width of the prepeak remain constant to a good approximation, whereas the main peak shifts slightly to higher $Q$ due to the increase in density. By using the pressure as an additional external parameter, one can disentangle the effects of density and temperature. We have thus measured the isothermal and isochoric evolutions of the $S(Q)$ of MFA. The results for the $T=285 \mathrm{~K}$ isotherm (liquid state) are displayed in the inset of fig. 1. For a density increase of about $10 \%$, the main peak moves to higher $Q$, as expected, whereas the prepeak appears remarkably constant in position, width, and intensity. The same features are also observed for the $T=150 \mathrm{~K}$ isotherm in the glassy state. A quite different behavior is obtained in the evolution of $S(Q)$ with temperature at a constant density of $1.3 \mathrm{gr} / \mathrm{cm}^{3}$ : as shown in fig. 1, the main peak remains roughly constant, which indicates no significant modification of the short-range order and local molecular packing, but the intensity of the prepeak increases by a factor of two when the temperature is decreased by $90 \mathrm{~K}$. The fact that the prepeak in MFA and MTOL seems to be only temperature dependent, together with the absence of prepeak in the $S(Q)$ of liquid and glassy TOL at $73 \mathrm{~K}$ [7], suggests that the prepeak is a consequence of hydrogen bonding interactions.

"Prepeaks" (or FSDP) are commonly observed in the $S(Q)$ 's of network glasses and are usually interpreted as the signature of a structure in real space that has a typical length greater than the first shell of neighbors [4]. Recent computer simulation studies support the idea that such an "intermediate- 
range order" originates from angular constraints in the topology of the random network formed by tetrahedrally coordinated atoms [8]. Despite the apparent similarity in the diffraction pattern, this explanation cannot be transposed to MFA and MTOL. As already stressed, these latter liquids are fragile. The H-bonding interactions are not dominant enough in their case to generate a continuous network nor to change the extreme fragility that is typical of the whole series of substituted aromatic liquids (as a fragility indicator, note that the parameter D of the Vogel-Tammann-Fulcher equation is between 3 and 6). Quite the contrary, network-forming systems are strong (e.g., $\mathrm{SiO}_{2}$ ) or intermediate (e.g. $\mathrm{ZnCl}_{2}$ ) glassformers. Several characteristic features of the prepeak are also very different in network glasses and in the present case. In MFA and MTOL the position of the prepeak is at lower $Q$ than it is in the network systems $\left(Q_{p p}=0.5 \AA^{-1}\right.$ compared to $\left.1-2 \AA^{-1}\right)$. The pressure dependences, as well as the temperature dependences, are also at variance since unlike in the vitreous $\mathrm{SiO}_{2}$ [9] the prepeaks in MFA and MTOL are mainly dominated by temperature and not by density changes. Moreover, while the main peak is sensitive to density changes and shifts to higher $Q$ by compressing or cooling, the prepeak position and its full width at half maximum are constant over a wide temperature and density range, from the stable liquid down to the glassy state. The inset of fig. 2 shows that the temperature dependence of the prepeak intensity in the supercooled liquid is more pronounced than the normal Debye-Waller dependence shown in the glassy state, and this is independent of the thermal treatment. Similar conclusions have been drawn from previous X-ray experiments at normal pressure [10].

Due to the low value of $Q_{p p}$ (one third of that of the main peak) and to the peculiar $\mathrm{T}$ and $\mathrm{P}$ dependences, the existence of the prepeak indicates a spatial organization of the molecules that goes beyond the usual short-range liquid order. However, its origin cannot be explained by pseudo-crystalline models [11] for several reasons. Firstly, the prepeak appears in a reversible way at temperatures above the melting point and persists in the stable liquid state. Secondly, for some systems a Bragg peak can be found at $0.5 \AA^{-1}$ in the $S(Q)$ of the crystal but no prepeak is observed in the $S(Q)$ of the glassy state as it was found for TOL [7].

In disordered systems, one often argues that the position of a peak reflects some repetitive characteristic distance between structural units and that its width corresponds to a correlation length [12, 13]. In order to check the possibility of an increasing structural correlation length in the liquid, the $S(Q)$ 's of partially deuterated MTOL obtained at different temperatures and normal pressure have been normalized at the prepeak position (cf. fig. 2). It clearly appears that the half-width at half-maximum is independent of 
temperature ; it yields a typical length of $50 \AA$, but no increase is detected from above the melting temperature down to below $T_{g}$, i.e., over more than $200 \mathrm{~K}$.

We suggest an explanation for the origin of the prepeak that involves the existence of H-bond-induced heterogeneities or clusters with no significant growth of the typical length. In order to gain more insight, we have also carried out Monte-Carlo simulations of MTOL by using an effective OPLS potential developed by Jorgensen [14]. Monte-Carlo simulations have been performed in the isothermal-isobaric ensemble $(N, P, T)$ at different temperatures and pressures with a Metropolis sampling. The system consists of 768 molecules (more than $10^{4}$ sites) in a cubic cell ca $50 \AA$ on a side with periodic boundary conditions. Full details on the simulations will be provided in a forthcoming publication. After an equilibration period covering $10^{6}$ to $3.10^{6}$ configurations, the structural properties were averaged over the next $2.10^{6}$ to $4.10^{6}$ configurations. The total and some partial $S(Q)$ 's and radial distribution functions (RDF) at atmospheric pressure and room temperature are presented in figs. 3a and 3b. We have checked that the agreement between experiment and simulation is very good for the total $S(Q)$ [7]. The numerical simulations reveal the existence of a short-to-medium range order that shows up in the partial structure factors associated with the $\mathrm{NH}_{2}$ group, $S_{\mathrm{NN}}(Q)$ or $S_{\mathrm{NH}}(Q)$ : the peak in $S_{\mathrm{NN}}(Q)$ or $S_{\mathrm{NH}}(Q)$ has a position at $0.5 \AA^{-1}$, which corresponds to that of the prepeak in the total $S(Q)$; its behavior is the same as that observed in the experiments described above when temperature or density is independently changed [7], while an isothermal compression tends to bring the peripheric groups $\left(\mathrm{C}, \mathrm{H}, \mathrm{CH}_{3}\right)$ closer and affects weakly the structure factor of the centers of the molecules, $S_{\mathrm{CC}}(Q)$ [15]. When one replaces the $\mathrm{NH}_{2}$ and $\mathrm{CH}_{3}$ groups by $\mathrm{H}$ atoms in the configurations of fig. 3a, the structure of benzene is restored [16]: the packing of the benzene rings is the dominant effect at $Q$ 's above $1.0 \AA^{-1}$ and H-bonding effect is only illustrated at lower $Q$ 's. The two contributions are well separated in the particular case of MTOL and MFA giving rise to a prepeak in the total structure factor. Studying the center of the molecule and the aminogroup RDF's provides two levels of structural organization as presented in fig. $3 \mathrm{~b}$. The former RDF presents damped oscillations with a period of 5-6 $\AA$, about one molecular diameter, and is very similar to what is observed in simple Van der Waals liquids [15]. In the latter appear first the distance N-N of $3 \AA$, which is typical of the H-bond itself, and an oscillation at $12 \AA$ (and seemingly at $25 \AA$ too). This oscillation occurs with a typical period, which is the mean intercluster separation or the mean cluster diameter. By Fourier transform, this typical period gives rise in Q-space to the prepeak at $0.5 \AA^{-1}$. On the basis of geometric criteria for the H-bond, we have checked the number of molecules 
connected by H-bonds in a cluster. We have found a size distribution of these clusters between 2 to 10 molecules, whose averaged number increases when the temperature decreases while the largest size stays at 10 molecules. The steric exclusion between aromatic rings prevents the extension of a continuous H-bond network contrary to what occurs in intermediate H-bonded liquids such as small alcohol molecules [5, 12. These clusters can be refered as structural heterogeneities specific to the systems under study; they do not occur in TOL since there is no H-bond interaction, nor in propylene glycol [5] or ethanol [12] where the packing constraint is reduced. These clusters do not seemingly order themselves, which is supported by the experimental observation that the prepeak width remains essentially constant. Because of this, the fragile character of these liquids is not altered and remains similar to that of the van der Waals TOL or the m-Xylene where no prepeaks are observed.

The clusters observed here have little effect on the overall slowing down of the relaxations with temperature, since it is very similar for the whole series of substituted aromatic liquids, irrespective of the presence of a prepeak in the $S(Q)$. Therefore, they are presumably not related to the heterogeneities or domains recently discussed in the litterature [1], 2].

However, the presence of clustering phenomena might induce significant differences in the relaxation times associated with different probes. In the case of MFA, the response under a mechanical stress is faster (by a factor of 3) than that under an electrical stress [17]. In both cases, the relaxations have roughly the same $T$-dependence, and the relaxation functions are highly non-exponential with a Kolraush (stretching) exponent $\beta$ of 0.37 for shear or volume relaxations and 0.61 for dielectric relaxation [17] at the same temperature $T=210 \mathrm{~K}$. The non-exponential character of the relaxations which is at variance to what is observed for monohydric alcohols [3] is probably related to the absence of a 3-dimensional network and to the polydispersity and the limited size of the clusters. As suggested in ref. 3, the experimental observations could be rationalyzed by considering that the dielectric process is dominated by the overall motion of the clusters contrary to the mechanical relaxation. An other interpretation could arise from recent experiments [18 which suggest that the slowest Debye-type process observed by dielectric spectroscopy for 1-propanol does is not assigned to any structural relaxation but to distinct $\mathrm{H}$-bonding effects. These special features due to the H-bonding are obviously not expected in a van der Waals isostructural system like m-xylene or TOL. In the latter case, as indeed suggested by recent work [19], the dielectric relaxation mode would not be the slowest one. More work in this direction would be usefull to clarify the dynamical signature of the H-bond-induced clusters in fragile liquids. 
The authors are very grateful to Dr G. Tarjus and Prof. M. Descamps for very helpful and stimulating discussions and to Drs A. Boutin and R. Pellenq for their help in numerical simulations. The authors would like to thank $\mathrm{R}$. Millet from L.L.B. for his constant technical assistance and support during all the experiments and Mrs Ratovelomanana for the sample preparation. This work has benefit from the use of the neutron source of the reactor Orphée (LLB, CEA-CNRS, Saclay) and C94 and C98 Cray computers facilities from IDRIS (Orsay). 


\section{References}

[1] CICEROne M. T. et al., J. Chem. Phys., 104 (1996) 7210; SCHMIDTROHR K. and SPIESS H.W., Phys. Rev. Lett. 66 (1991) 3020; BOHMER R. et al., Europhys. Lett., 36 (1996) 55; SCHIENER B. et al., Science, 274 (1996) 752.

[2] KIRKPATRICK T. R. et al., Phys. Rev. A, 40 (1989) 1045; MEL'CUK A. I., Phys. Rev. Lett., 75 (1995) 2522; KIVELSON D. et al., PHYSICA A 219 (1995) 27; KIVELSON D. and TARJUS G., Prog. Theor. Phys. in press (1998).

[3] Floriano M. A. and ANGell C. A., J. Chem. Phys., 91 (1989) 2537; ANGELL C.A., in "Correlations and Connectivity", H. E. STANLEY, N. OSTROWSKY (eds.), Kluwer Acad. Publish. (1990).

[4] ELliOTT S. R., Nature, 354 (1991) 445; J. Phys. : Condens. Matter, 4 (1992) 7661.

[5] LEHENY R. et al., J. Chem. Phys., 105 (1996) 7783.

[6] ANGELL C. A., p.3 in "Relaxation in Complex Systems", NGAI K. L. and WRIGHT G. B. (eds.) Office of Naval Research, Washington, (1984); ALBA-SIMIONESCO C., FAN J. and ANGELL C. A., submitted to J. Chem. Phys., (1998).

[7] MORINEAU D. and ALBA-SIMIONESCO C., to be published.

[8] WILSON M. and MADDEN P., J. Phys. : Condens. Matter, 6 (1994) A151.

[9] SUGAi S. and ONODERA A., Phys. Rev. Lett., 77 (1996) 4210.

[10] DESCAMPS M. et al., Prog. Theor. Phys., 126 (1997) 207.

[11] GASKELL P. H., Materials Science and Technology, CAHN R. W. et al.,(ed.), 9 (1991) 175.

[12] SRINIVASAN A. et al., Phys. Rev. B, 53 (1996) 8172; FAYOS R. et al., Phys. Rev. Lett., 77 (1996) 3823.

[13] SAlmon P., Proc. R. Soc. Lond. A, 445 (1994) 351.

[14] JORGEnSEN W. et al., J. of Comput. Chem., 14 (1993) 206.

[15] MORINEAU D. et al., Molec. Sim., 20 (1997) 95. 
[16] BARTSCH E. et al., Ber. Bunsenges. Phys. Chem., 89 (1985) 147; Chem. Phys., 169373 (1993).

[17] CUtroni M. et al., J. Phys.: Condens. Matter, 65283 (1994); ALBASIMIONESCO C., unpublished results.

[18] HANSEN C.et al., J. Chem. Phys., 107 (1997) 1086.

[19] DOSS A. et al., J. Chem. Phys., 107 (1997) 1740. 
Figure captions

Figure 1: Total static structure factor $S(Q)$ of MFA $\left(T_{g}=170 \mathrm{~K}\right.$, not known $T_{m}$ ) along the isochore $\rho=1.3 \mathrm{gr} / \mathrm{cm}^{3}$, at $T=195 \mathrm{~K}, 0.1 \mathrm{MPa}$ (circles) and $T=285 \mathrm{~K}, 260 \mathrm{MPa}$ (squares). The difference between the 2 spectra (triangles) is only sensitive to the prepeak intensity. The inset shows the same fonction along the isotherm $\mathrm{T}=285 \mathrm{~K}$ at two pressures $0.1 \mathrm{MPa}$ (circles) and $260 \mathrm{MPa}$ (squares) corresponding to a density change of $10 \%$. The difference between the 2 spectra (triangles) only shows a shift of the main peak position.

Figure 2: Reduced structure factor $\Delta S(Q, T) / \Delta S(Q=0.5, T)$ of the partially deuterated liquid MTOL $\left(\mathrm{C}_{6} \mathrm{D}_{4} \mathrm{CD}_{3} \mathrm{NH}_{2}\right)$ at $0.1 \mathrm{MPa}$ for different T's corresponding to the points in the inset. It is obtained by subtracting $S(Q, T=310 \mathrm{~K})$ from $S(Q, T)$ and normalizing at the prepeak position $Q_{p p}=0.5 \AA^{-1}$. Note that the width and the position of the prepeak are constant, whereas the main peak changes with $T$. The inset shows $\Delta S(Q=0.5, T)$ as a function of $T$.

Figure 3: Results of Monte-Carlo simulation of MTOL at $T=291 \mathrm{~K}$ and $0.1 \mathrm{MPa}$. a) Total structure factor $S(Q)$ (circles), partial structure factor $S_{\mathrm{NN}}(Q)$ (full lines) and center-to-center structure factor $S_{\mathrm{cc}}(Q)$ (dotted lines). Also displayed (squares) the $S(Q)$ for a pseudo-benzene obtained by replacing all $\mathrm{NH}_{2}$ and $\mathrm{CH}_{3}$ groups by $\mathrm{H}$ atoms in the configurations of MTOL. The low $Q$ residual shoulder appearing in the partial $S(Q)$ and for the pseudo-benzene is due to the Fourier transformation procedure. b) N-N partial RDF (squares) and center-to-center partial RDF (rings) presenting oscillations with two different associated periods. 
Fig. 1

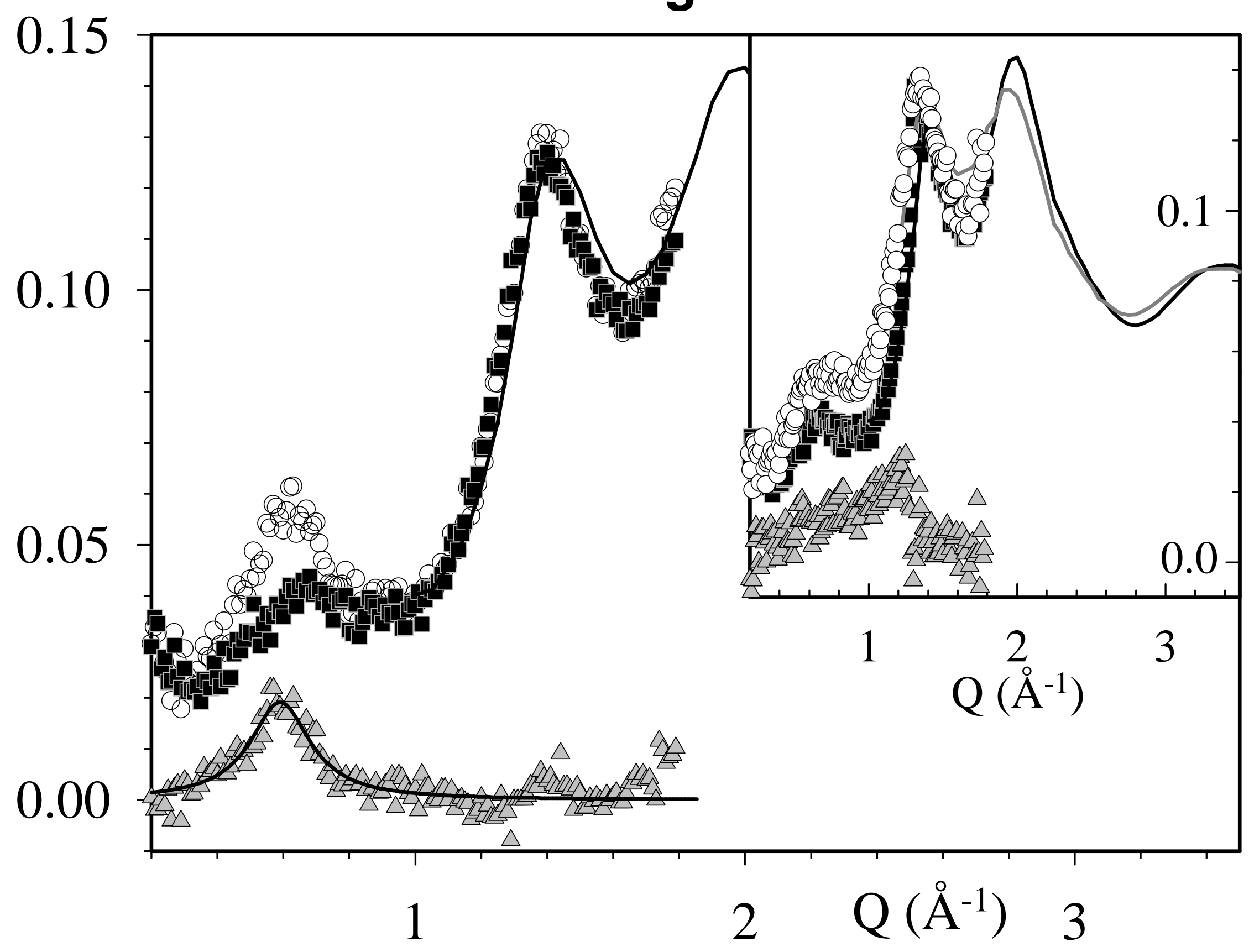


Fig. 2

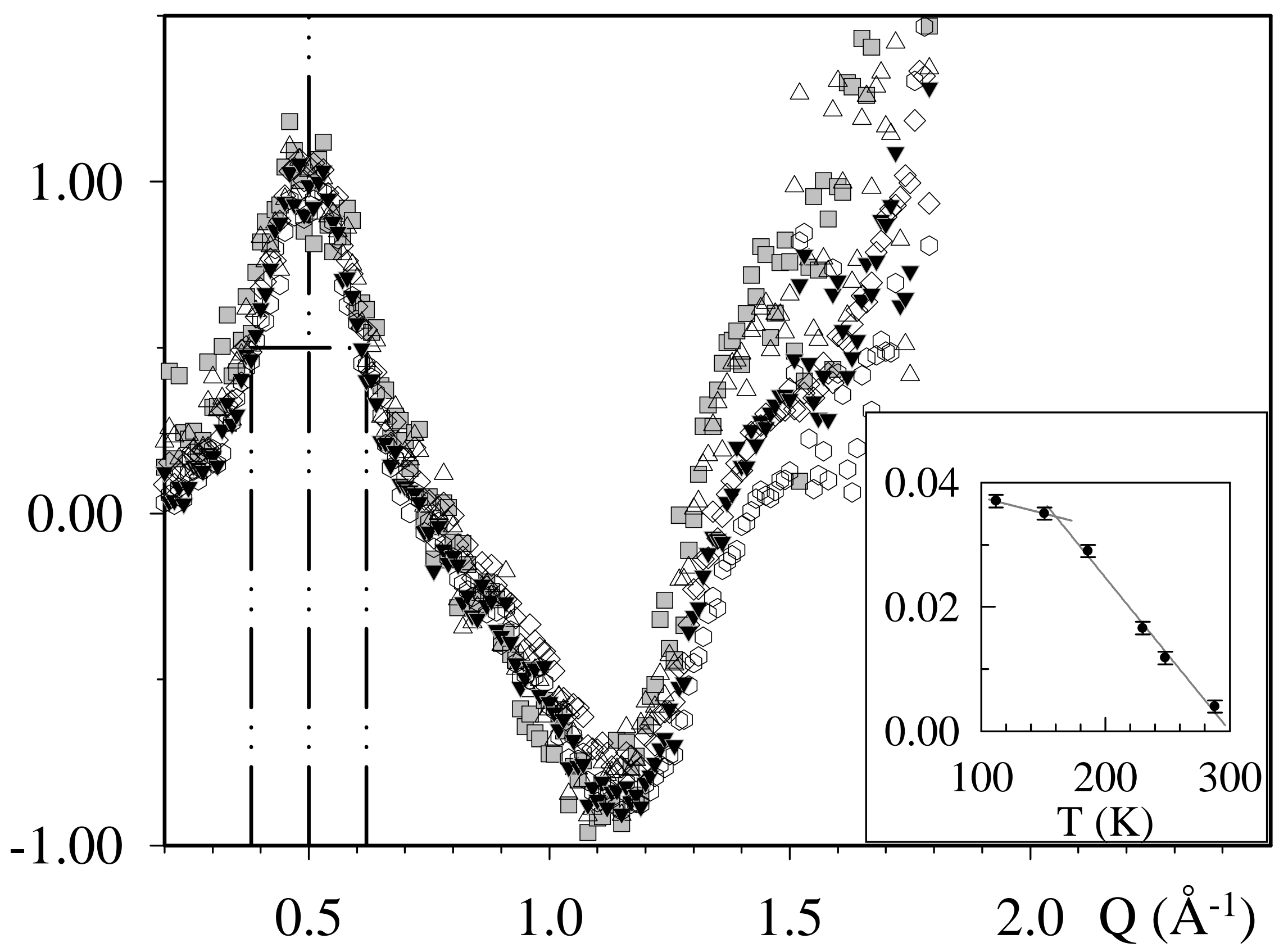


Fig. 3 a

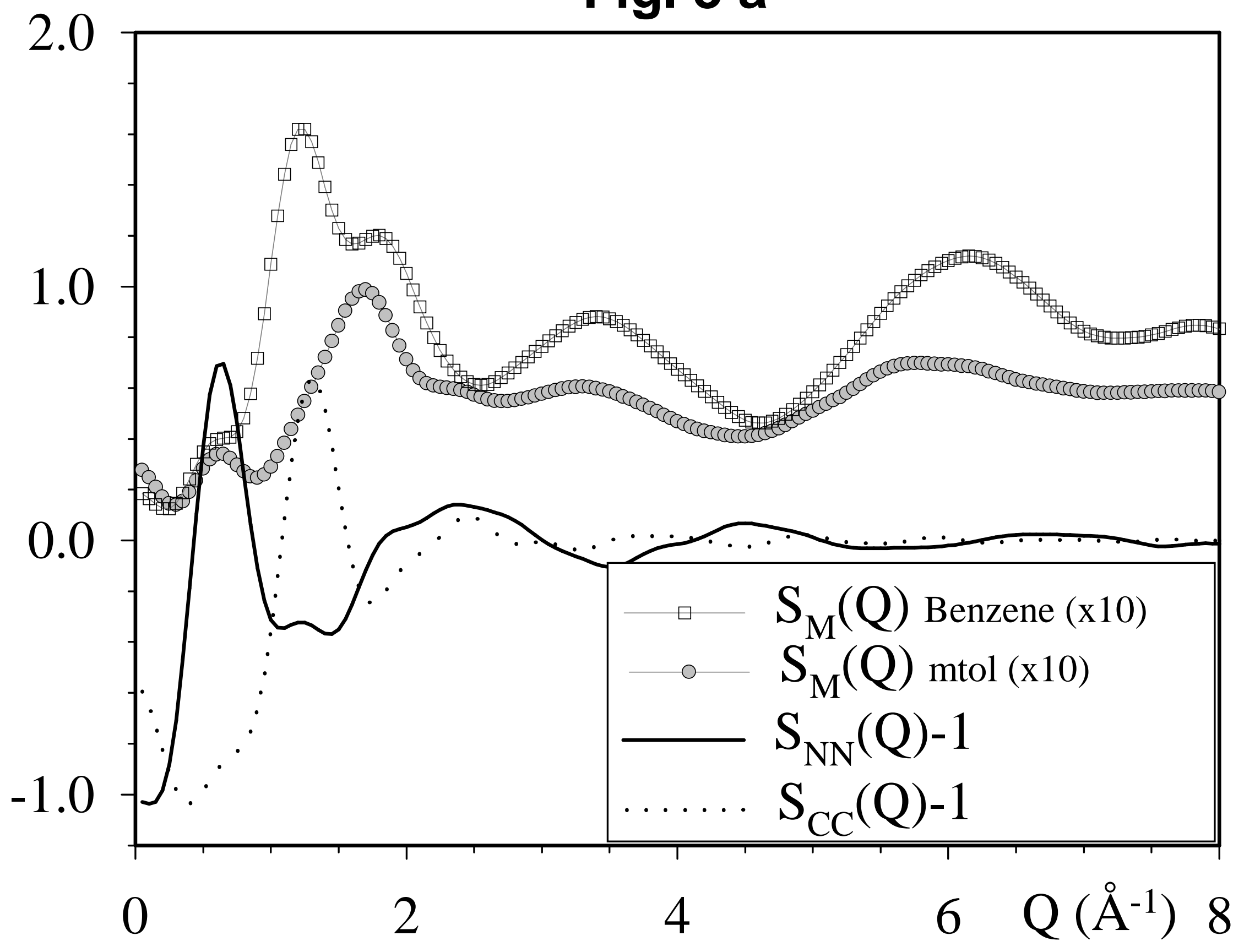


Fig. 3 b

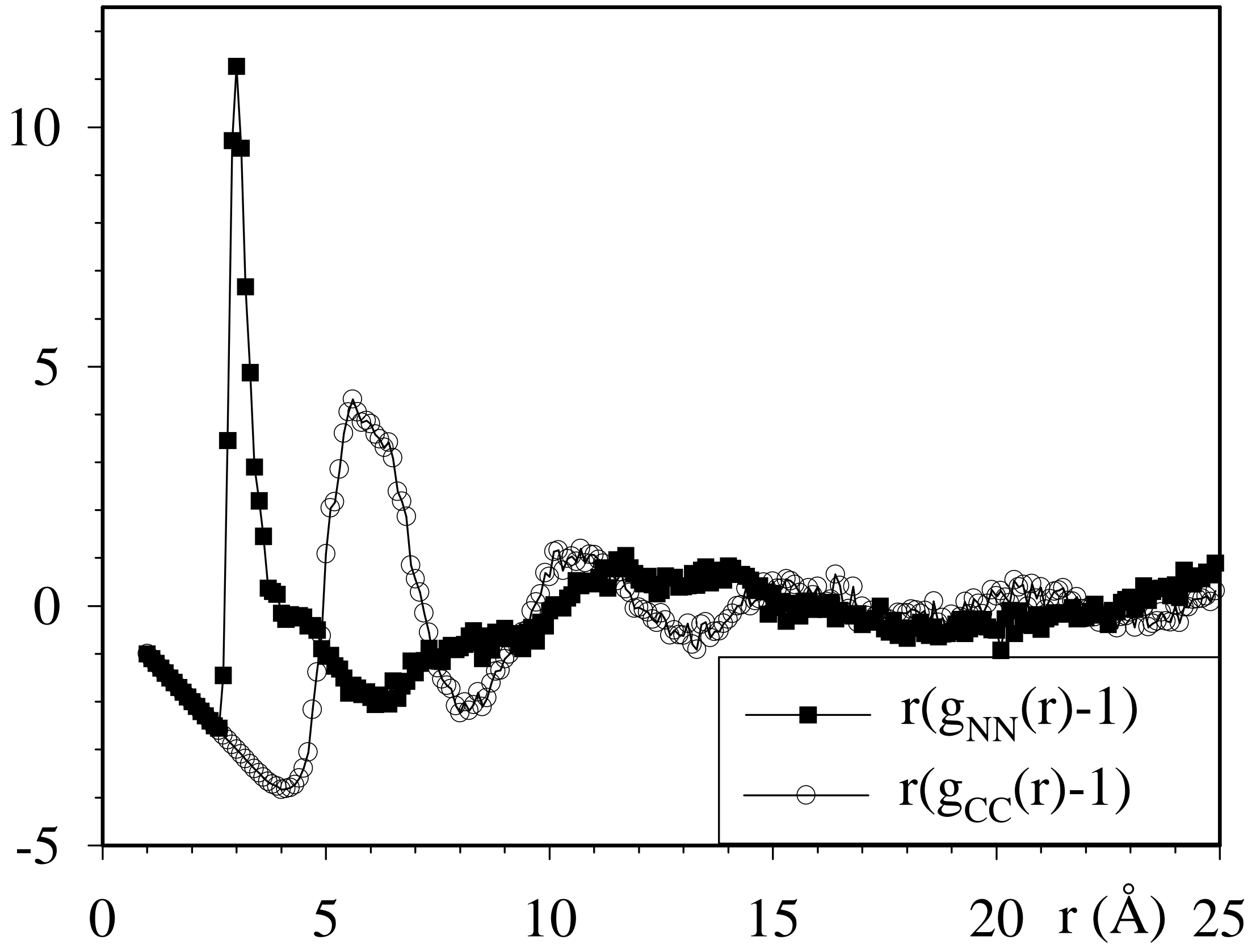

